\title{
Persistent frequent subclinical seizures and memory impairment after clinical remission in smoldering limbic encephalitis.
}

\author{
AUTHOR(S): \\ Kanazawa, Kyoko; Matsumoto, Riki; Shimotake, Akihiro; \\ Kinoshita, Masako; Otsuka, Akiko; Watanabe, Osamu; \\ Tanaka, Keiko; Takahashi, Ryosuke; Ikeda, Akio
}

\section{CITATION:}

Kanazawa, Kyoko ... [et al]. Persistent frequent subclinical seizures and memory impairment after clinical remission in smoldering limbic encephalitis.. Epileptic disorders 2014, 16(3): 312-317

\section{ISSUE DATE:}

2014-09

URL:

http://hdl.handle.net/2433/198597

\section{RIGHT:}

(c) John Libbey Eurotext; 許諾条件により本文ファイルは2015-10-01に 公開. 


\title{
Persistent frequent subclinical seizures and memory impairment after clinical remission in smoldering limbic encephalitis
}

\author{
Kyoko Kanazawa ${ }^{1}$, Riki Matsumoto ${ }^{2}$, Akihiro Shimotake ${ }^{1}$, \\ Masako Kinoshita ${ }^{3}$, Akiko Otsuka ${ }^{4}$, Osamu Watanabe ${ }^{5}$, \\ Keiko Tanaka ${ }^{6}$, Ryosuke Takahashi ${ }^{1}$, Akio Ikeda ${ }^{2}$ \\ ${ }^{1}$ Department of Neurology \\ ${ }^{2}$ Department of Epilepsy, Movement Disorders and Physiology, Kyoto University \\ Graduate School of Medicine, Kyoto \\ ${ }^{3}$ Department of Neurology, Utano National Hospital, Utano \\ ${ }^{4}$ Department of Respiratory Medicine, Kyoto University Graduate School of Medicine, \\ Kyoto \\ ${ }^{5}$ Department of Neurology and Geriatrics, Kagoshima University Graduate School \\ of Medical and Dental Sciences, Kagoshima \\ ${ }^{6}$ Department of Neurology, Kanazawa Medical University, Kanazawa, Japan
}

Received November 24, 2013; Accepted May 07, 2014

Correspondence:

Riki Matsumoto, Akio Ikeda

54 Shogoin-Kawaharacho,

Sakyo-ku,

Kyoto-shi,

Kyoto 606-8507, Japan

<matsumot@kuhp.kyoto-u.ac.jp>

<akio@kuhp.kyoto-u.ac.jp>

\begin{abstract}
Aim. To delineate a possible correlation between clinical course and EEG abnormalities in non-infectious "smoldering" limbic encephalitis. Methods. Long-term clinical data, including video-EEG monitoring records, were analysed in two patients. Results. The two patients were positive for anti-voltage-gated potassium channel complex antibody and unspecified antineuronal antibody, respectively. The latter patient had small cell lung carcinoma. Both patients had memory impairment and clinical seizures. EEG showed frequent subclinical seizure patterns in the bilateral temporal regions. Subclinical seizure patterns and memory impairment persisted over one to two years after clinical seizure remission. Therapy (prednisolone and chemoradiation in the two patients, respectively) resulted in decreased occurrence of subclinical seizure patterns and memory improvement. Conclusions. EEG seizure patterns may persist years after clinical seizure remission in "smoldering" limbic encephalitis and lead to memory impairment.
\end{abstract}

Key words: EEG seizure pattern, memory impairment, smoldering limbic encephalitis, voltage-gated potassium-channel complex antibody, antineuronal antibody, epilepsy

Recent studies of non-infectious limbic encephalitis have reported a number of syndromes associated with various autoantibodies, such as anti-voltage-gated potassium channel (VGKC) complex antibodies (Vincent et al., 2004). Although autoantibodies should be sought 
in non-infectious limbic encephalitis (Pruss et al., 2010), the list of relevant biomarkers or antibodies is so far incomplete. Negative results do not exclude paraneoplastic or autoimmune disorders, and antineuronal antibodies without specific antigen characterisation are sometimes positive based on immunohistochemical analysis. This result would justify immunotherapy in individual patients, and further research on these cases may reveal new antigens in the future (Zuliani et al., 2012).

To our knowledge, despite many reports on immunemediated limbic encephalitis, chronological change of EEG abnormalities, along with anatomo-functional imaging findings over a period of years, related to a "smoldering" clinical course, even after clinical seizure remission, has not been described in detail in the literature. In the present study, we aimed to delineate a possible correlation in two welldocumented patients with non-infectious smoldering limbic encephalitis. We previously reported a case of limbic encephalitis associated with anti-VGKC complex antibodies, to which we referred to as "smoldering limbic encephalitis" (Nakaoku et al., 2013). In the present study, we describe two patients with "smoldering limbic encephalitis" due to the presence of limbic encephalitis with persisting chronic symptoms, such as memory impairment and personality change, as well as other abnormalities such as EEG seizure patterns and/or residual increased glucose metabolism, persisting for more than a year, even after clinical seizure remission. We specifically chose the word "smoldering" due to the suggestion of residual active chronic inflammation based on increased glucose metabolism on 18F-fluorodeoxyglucose-positron emission tomography (FDG-PET) in at least one of the two patients, whereas epilepsy patients, in general, demonstrate glucose hypometabolism.

\section{Materials and methods}

We retrospectively analysed clinical data of two patients with immune-mediated limbic encephalitis, for whom long-term follow-up data with video-EEG records were available.

Patient 1 was a 62-year-old man with non-infectious smoldering limbic encephalitis without autoimmune comorbidities, such as systemic lupus erythematoses. Patient 2 was a 60 -year-old man with paraneoplastic limbic encephalitis. Revised Hasegawa's Dementia Scale (Imai and Hasegawa, 2005) was performed for Patient 1, and Mini-Mental State Examination (MMSE) for Patient 2. The Wechsler Adult Intelligence Scale-Revised (WAIS-R) and Wechsler Memory Scale-Revised (WMS-R) were also performed on three and two occasions for Patients 1 and 2, respectively.
Laboratory examinations, including autoantibody tests, cerebrospinal fluid (CSF) analysis, magnetic resonance imaging (MRI), and FDG-PET, were performed. Video- and routine EEGs were recorded on multiple occasions over the clinical course of 32 months for Patient 1, and 58 months for Patient 2. Patient 1 was treated with prednisolone and Patient 2 with chemoradiation.

\section{Results}

Patient 1 was positive for anti-VGKC complex antibodies (in July 2009), but negative for anti-Hu, Yo and Ri antibodies. He had a generalised tonic-clonic seizure, followed by gradual memory impairment in November 2008. He was initially given carbamazepine (CBZ), at $200 \mathrm{mg} /$ day, which was later substituted for valproate (VPA), at 1,200 mg/day, due to skin rash caused by CBZ. Although no seizure recurred, memory impairment worsened (VPA blood concentration: $59.0 \mu \mathrm{g} / \mathrm{mL}$ ). He also had a change in personality and became very irritable.

In July 2009, although the WAIS-R showed preserved intelligence, the WMS-R showed moderate memory impairment (see figure 1 and table 1). The CSF analysis was normal: $0 / \mathrm{mm}^{3}$ cells; $27.3 \mathrm{mg} / \mathrm{dL}$ protein; $57 \mathrm{mg} / \mathrm{dL}$ glucose; and an IgG index of 0.45 (reference value: 0.34-0.85). The bilateral mesial temporal lobes showed increased volume and high intensity signal abnormality on MRI fluid-attenuated inversion recovery (FLAIR) images, and increased glucose metabolism on FDGPET. Video-EEG showed 52 subclinical EEG seizure patterns, independently in the left and right anterior temporal regions over two days, and interictal transient sharp waves in the right temporal region. After methylprednisolone pulse therapy ( $1 \mathrm{~g} /$ day for three days), memory and irritability gradually improved.

Follow-up WAIS-R and WMS-R, 10 months later in May 2010, showed improved intelligence and memory. The hippocampi were atrophic on the right, and still hypertrophic on the left, on MRI. The bilateral mesial temporal lobes showed residual, though less prominent, high glucose metabolism on FDG-PET. Video-EEG showed bilateral independent subclinical EEG seizure patterns 10 times over two days, and interictal sharp waves in the left and right temporal regions, independently. After a second methyl-prednisolone pulse therapy, memory impairment remained stable and irritability improved.

His irritability, as well as follow-up WAIS-R and WMS-R scores, remained stable in July 2011. The bilateral hippocampi were atrophic on MRI. The bilateral mesial temporal lobes showed residual high glucose metabolism on FDG-PET, although this had further improved. A third methyl-prednisolone pulse therapy 


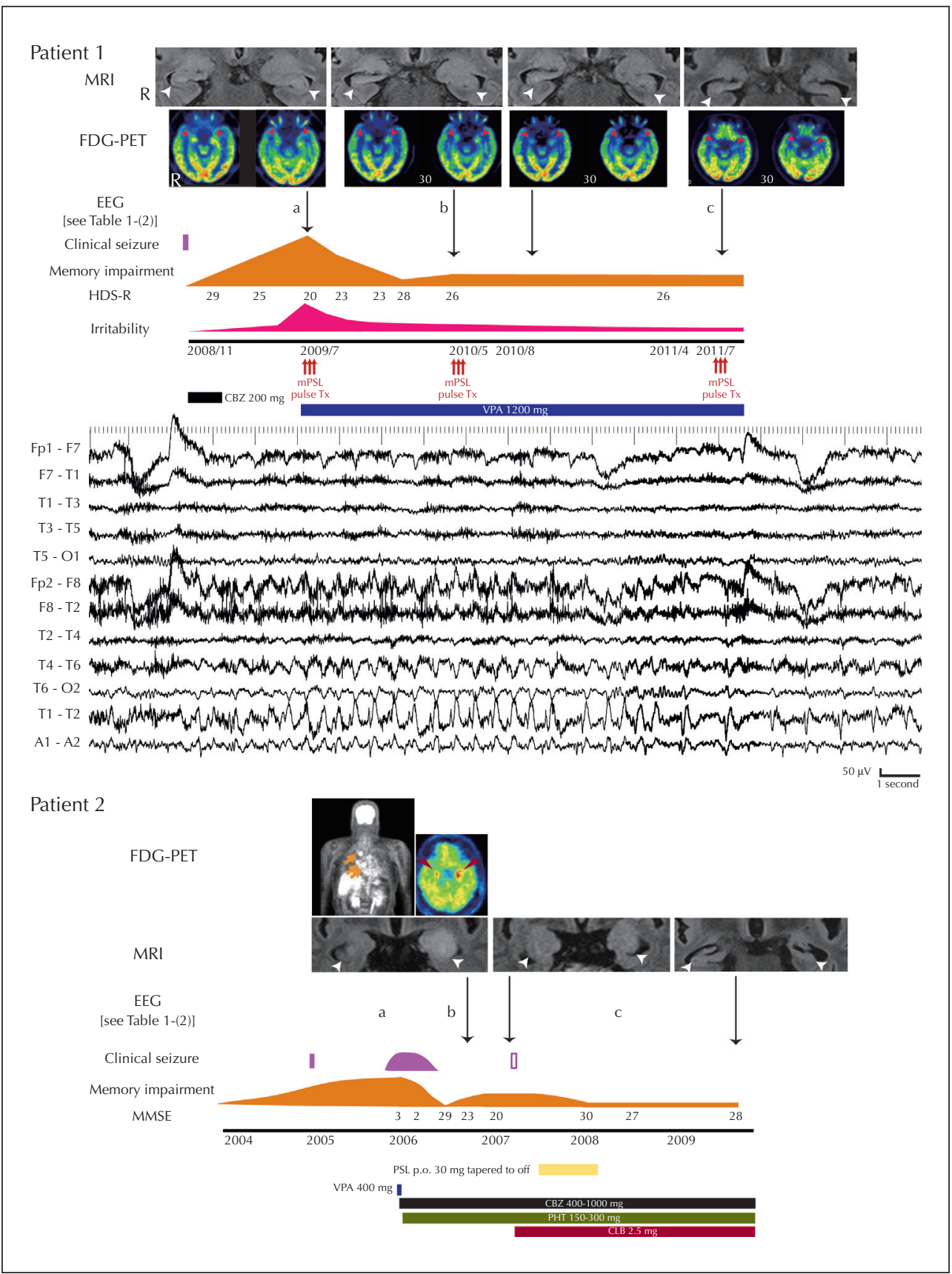

Figure 1. Clinical course, imaging findings, and ictal EEG pattern.

The small filled purple rectangles for Patients 1 and 2 indicate isolated generalised seizures, and the small open rectangle in Patient 2 indicates an isolated complex partial seizure. The shaded purple area for Patient 2 indicates frequency of simple partial and secondary generalised tonic-clonic seizures. White arrowheads in the MR image (FLAIR) in Patients 1 and 2 indicate abnormal hippocampi. Red arrowheads in the FDG-PET image in Patients 1 and 2 indicate increased uptake in the mesial temporal lobes. Orange arrows in the FDG-PET image in Patient 2 indicate increased uptake in the mediastinum and subcarinal lymph nodes due to small cell lung carcinoma. Ictal EEG pattern as a subclinical seizure in the right temporal region is shown in Patient 1. Patient 1 had no clinical seizures except one generalised seizure at the beginning of his clinical course. EEG: bipolar montage; sampling rate: $200 \mathrm{~Hz}$; high frequency filter: $60 \mathrm{~Hz}$; TC: $0.3 \mathrm{sec}$.

CBZ: carbamazepine; CLB: clobazam; FDG-PET: ${ }^{18}$ F-fluorodeoxyglucose-positron emission tomography; FLAIR: fluid-attenuated inversion recovery; HDS-R: Revised Hasegawa's Dementia Scale (Imai and Hasegawa, 2005) (consisting of nine simple questions with a maximum score of 30; a widely accepted scale not only for clinical use but also for epidemiological surveys of cognition in Japan $[\leq 21$ regarded as cognitive decline]); MMSE: Mini-Mental State Examination; mPSL: methylprednisolone; PHT: phenytoin; p.o.: per oral; PSL: prednisolone; R: right; Tx: therapy; VPA: valproate. 
Table 1. Chronological change of psychometric examination.

\begin{tabular}{|c|c|c|c|c|}
\hline & \multicolumn{4}{|c|}{ Neuropsychological assessment } \\
\hline \multirow{11}{*}{ Patient 1} & & July 2009 & May 2010 & July 2011 \\
\hline & WAIS-R: & & & \\
\hline & VIQ & 105 & 113 & 111 \\
\hline & PIQ & 107 & 113 & 113 \\
\hline & FIQ & 106 & 114 & 113 \\
\hline & WMS-R: & & & \\
\hline & Verbal memory & 63 & 88 & 79 \\
\hline & Visual memory & 68 & 97 & 110 \\
\hline & General memory & 59 & 90 & 88 \\
\hline & Attention & 112 & 125 & 71 \\
\hline & Delayed memory & $<50$ & 65 & 68 \\
\hline \multirow{11}{*}{ Patient 2} & & October 2006 & April 2007 & \\
\hline & WAIS-R: & & & \\
\hline & VIQ & 86 & 83 & \\
\hline & PIQ & 101 & 96 & \\
\hline & FIQ & 93 & 89 & \\
\hline & WMS-R: & & & \\
\hline & Verbal memory & 60 & 74 & \\
\hline & Visual memory & 81 & 50 & \\
\hline & General memory & 61 & 61 & \\
\hline & Attention & 101 & 92 & \\
\hline & Delayed memory & 50 & $<50$ & \\
\hline
\end{tabular}

FIQ: full intelligence quotient; PIQ: performance intelligence quotient; VIQ: verbal intelligence quotient; WAIS-R: Wechsler Adult Intelligence Scale-Revised; WMS-R: Wechsler Memory Scale-Revised.

was given and his memory impairment and irritability have remained stable to date.

Patient 2 was negative for anti-VGKC complex, $\mathrm{Hu}$, Yo, Ri, CV2, Tr, Ma2, and amphiphysin antibodies, but positive for an unspecified antineuronal antibody (immunohistochemical staining using the patient's serum with biotin-labelled antihuman IgG and avidinbiotin complex [Tanaka et al., 1994] gave rise to a diffuse staining pattern associated with the neuropil, rather suggestive of a cell surface antibody). His memory gradually declined from 2004, followed by the development of complex partial and generalised tonicclonic seizures in 2005. During the initial work-up in January 2006, he stared with a blank look and hardly spoke. MMSE score was 3/30. He continued to have impaired orientation and abnormal behaviour even after he was able to have a conversation. MRI was normal. EEG showed background slowing $(6-7 \mathrm{~Hz})$ without paroxysmal discharges (see figure 1 and table 2). It was therefore assumed that he most likely had diffuse encephalopathy at that time. He continued to have seizures, simple partial and secondary generalised tonic-clonic, with VPA treatment at $400 \mathrm{mg}$ /day. The CSF analysis was normal: $3 / \mathrm{mm}^{3}$ cells; $37 \mathrm{mg} / \mathrm{dL}$ protein; $59 \mathrm{mg} / \mathrm{dL}$ glucose; and an IgG index of 0.58 . Seizure control improved with phenytoin (PHT) at $150 \mathrm{mg} /$ day and CBZ at $900 \mathrm{mg} /$ day, and his MMSE score improved to $29 / 30$.

His memory impairment recurred in October 2006. The left hippocampus showed increased volume and high intensity signal abnormality on MRI (FLAIR). The bilateral mesial temporal lobes showed increased glucose metabolism on FDG-PET, as well as in the mediastinum and subcarinal lymph nodes. Videoand routine EEGs showed three to four subclinical seizure patterns per hour in the left and right temporal regions, independently, and interictal intermittent rhythmic delta activities in the left temporal region.

Following a diagnosis of small cell lung carcinoma (SCLC), he underwent chemoradiation therapy (cisplatin+etoposide/60 Gy, followed by carboplatin+irinotecan, and then amrubicin). Seizures remitted after the addition of clobazam (CLB) at $2.5 \mathrm{mg} /$ day (blood concentration: $14.9 \mu \mathrm{g} / \mathrm{mL}$ PHT; $6.1 \mu \mathrm{g} / \mathrm{mL}$ CBZ; $13 \mathrm{ng} / \mathrm{mL}$ CLB; $345 \mathrm{ng} / \mathrm{mL}$ desmethylCLB), and his memory improved and stabilised. Follow-up MRI showed increased volume and high intensity signal abnormality also in the right hippocampus (February 2007), followed by bilateral hippocampal atrophy (June 2009). EEG seizure patterns decreased to $1 / 30$ minutes. 
Table 2. Chronological change of EEG.

\begin{tabular}{|c|c|c|c|c|}
\hline & $\begin{array}{c}\text { Record date } \\
\text { (refer to figure 1) }\end{array}$ & Recording time & Interictal paroxysmal discharge & Seizure pattern \\
\hline Patient 1 & $\begin{array}{l}\mathrm{a} \\
\mathrm{b} \\
\mathrm{c}\end{array}$ & $\begin{array}{l}2 \text { days } \\
2 \text { days } \\
2 \text { days }\end{array}$ & $\begin{array}{l}\text { transient sharp waves } \\
\text { R FT } \\
\text { sharp waves } \\
\text { R or L FT } \\
\text { transient sharp waves } \\
\text { R Fp }\end{array}$ & $\begin{array}{l}\mathrm{L}=\text { twice } \\
\mathrm{R}=50 \text { times } \\
\mathrm{L}=\text { once } \\
\mathrm{R}=9 \text { times } \\
\mathrm{L}=6 \text { times } \\
\mathrm{R}=4 \text { times }\end{array}$ \\
\hline Patient 2 & $\begin{array}{l}\mathrm{a} \\
\mathrm{b}\end{array}$ & $\begin{array}{l}30 \text { minutes } \\
7.5 \text { hours } \\
30 \text { minutes } \\
30 \text { minutes }\end{array}$ & $\begin{array}{l}\text { none } \\
\text { TIRDA } \\
\text { L mT } \\
\text { none } \\
\text { sharp wave } \\
\text { L FT }\end{array}$ & $\begin{array}{l}\text { none } \\
L=29 \text { times } \\
R=\text { none } \\
L=\text { none } \\
R=\text { twice } \\
L=\text { once } \\
R=\text { none }\end{array}$ \\
\hline
\end{tabular}

FT: fronto-temporal; Fp: fronto-polar; L: left; mT: mid-temporal; R: right; TIRDA: temporal intermittent rhythmic delta activity.

His clinical course was complicated with radiation pneumonitis due to radiotherapy for SCLC. Prednisolone at $30 \mathrm{mg} /$ day was started in July 2007, which was subsequently tapered off. Cancer recurred with hepatic metastasis in April 2008, without further memory deterioration. He died in August 2009.

\section{Discussion}

Autoimmune pathophysiology such as limbic encephalitis has been recently described in relation to both acute (Buckley et al., 2001; Vincent et al., 2004; Dalmau et al., 2007; Quek et al., 2012) and chronic epileptogenicity (Brenner et al., 2013). Autoantibodies such as anti-VGKC complex antibodies and anti-N-methyl D-aspartate (NMDA) receptor antibodies have been reported to be related to seizures mostly with an acute clinical course. The two patients manifested a clinically "smoldering" course with EEG seizure patterns persisting for one to two years even after clinical seizure remission. This phenomenon has not been well documented in the literature, at least for immune-mediated limbic encephalitis.

In the present study, Patients 1 and 2 were positive for anti-VGKC complex antibody and for an unspecified antineuronal antibody, respectively. Their clinical courses correlated with imaging abnormality in the bilateral mesial temporal lobes. Memory impairment was predominant, while clinical seizures were infrequent, despite very frequent EEG seizure patterns.
Even after clinical seizure remission, EEG seizure patterns persisted for one to two years, and thus might have contributed to memory impairment, at least partly, in addition to limbic encephalitis itself.

Video-EEG has been reported to be useful in the early diagnosis of acute anti-NMDA receptor encephalitis, as confirmation of NMDA receptor antibodies takes several weeks (Dericioglu et al., 2013; Di Capua et al., 2013; Tan et al., 2013). The present study suggests that video-EEG, even over only one to two days, could also be a clinically useful means to evaluate and follow up a "smoldering" clinical state, and possibly limbic encephalitis itself. Video-EEG could therefore be considered when chronic symptoms, such as memory impairment and personality change, persist even after clinical seizure remission, and the results of laboratory examinations other than video-EEG do not provide a clear explanation for the persistent symptoms.

With regards to immunotherapy for the treatment of epilepsy, in general, plasmapheresis and steroid therapy for Rasmussen encephalitis have been well established, although the immunological basis of the treatment remains to be determined. With regards to immunotherapy for the treatment of epilepsy patients with positive autoantibodies, the response rate to immunotherapy in patients with exclusive or predominant seizure presentation, in whom an autoimmune aetiology was suspected (with a median history of seizure activity of five months [range: 3 weeks to 12 years]), was reported to be $81 \%$, and $67 \%$ 
patients were seizure-free (Quek et al., 2012). In NMDA receptor encephalitis, second-line immunotherapy (rituximab, cyclophosphamide) has been reported to be effective when first-line therapies (steroids, intravenous immunoglobulin, plasmapheresis) fail (with a time between symptom onset and initiation of immunotherapy of 18 days according to Ikeguchi et al. [2012], and 21 days in children and 28 days in adults according to Titulaer et al., [2013]). In the present study, prednisolone in Patient 1 and chemoradiation in Patient 2 resulted in decreased frequency of EEG seizure patterns and memory improvement. Improvement in Patient 2 may have also reflected a favourable effect of oral prednisolone therapy given for radiation pneumonitis.

As a limitation of the present study, the immunological testing was incomplete; although different kinds of autoantibodies were tested, it remains possible that our patients were positive for other untested autoantibodies.

In conclusion, for a chronic course persisting to a milder degree, i.e. "smoldering", non-infectious limbic encephalitis should be considered as a possible diagnosis when bilateral independent interictal epileptiform discharges and EEG seizure patterns are observed in elderly patients. These patients need appropriate diagnosis and treatment in terms of autoimmunity. $\square$

\section{Acknowledgements and disclosures.}

The authors thank Prof. Angela Vincent (University of Oxford, UK) for measuring anti-VGKC complex antibodies in Patient 1. The study was partly supported by the Grants-in-Aid for Scientific Research (C23591273 to RM, 23591275 to AI) from the Japan Ministry of Education, Culture, Sports, Science and Technology (MEXT), and the Research Grant to AI (22A-6) (2320160) for Nervous and Mental Disorders from the Ministry of Health and Welfare.

None of the authors have any conflicts of interests to disclose.

\section{References}

Brenner T, Sills GJ, Hart Y, et al. Prevalence of neurologic autoantibodies in cohorts of patients with new and established epilepsy. Epilepsia 2013; 54:1028-35.

Buckley C, Oger J, Clover L, et al. Potassium channel antibodies in two patients with reversible limbic encephalitis. Ann Neurol 2001; 50: 73-8.
Dalmau J, Tuzun E, Wu HY, et al. Paraneoplastic anti-Nmethyl-D-aspartate receptor encephalitis associated with ovarian teratoma. Ann Neurol 2007; 61: 25-36.

Dericioglu N, Vural A, Acar P, et al. Antiepileptic treatment for anti-NMDA receptor encephalitis: the need for videoEEG monitoring. Epileptic Disord 2013; 15: 166-70. doi: 10.1684/epd.2013.0566.

Di Capua D, Garcia-Ptacek S, Garcia-Garcia ME, Abarrategui B, Porta-Etessam J, Garcia-Morales I. Extreme delta brush in a patient with anti-NMDAR encephalitis. Epileptic Disord 2013; 15: 461-4. doi: 10.1684/epd.2013.0622.

Ikeguchi R, Shibuya K, Akiyama S, et al. Rituximab used successfully in the treatment of anti-NMDA receptor encephalitis. Intern Med 2012; 51:1585-9.

Imai Y, Hasegawa K. The Revised Hasegawa's Dementia Scale (HDS-R)- Evaluation of its usefulness as a screening test for dementia. Hong Kong Journal of Psychiatry 2005; 4: 20-4.

Nakaoku Y, Maki T, Kanazawa K, et al. A case of smoldering anti-leucine-rich glioma-inactivated 1 (LGI1) antibodyassociated limbic encephalitis with faciobrachial dystonic seizure. Rinsho Shinkeigaku 2013; 53: 706-11.

Pruss H, Dalmau J, Harms L, et al. Retrospective analysis of NMDA receptor antibodies in encephalitis of unknown origin. Neurology 2010; 75: 1735-9.

Quek AM, Britton JW, McKeon A, et al. Autoimmune epilepsy: clinical characteristics and response to immunotherapy. Arch Neurol 2012; 69: 582-93.

Tan YL, Tan K, Tan NC. Antiepileptic treatment for anti-NMDA receptor encephalitis: the need for video-EEG monitoring. Epileptic Disord 2013; 15: 468. doi: 10.1684/epd.2013.0620.

Tanaka K, Tanaka M, Onodera O, Igarashi S, Miyatake T, Tsuji $S$. Passive transfer and active immunization with the recombinant leucine-zipper (Yo) protein as an attempt to establish an animal model of paraneoplastic cerebellar degeneration. J Neurol Sci 1994; 127: 153-8.

Titulaer MJ, McCracken L, Gabilondo I, et al. Treatment and prognostic factors for long-term outcome in patients with anti-NMDA receptor encephalitis: an observational cohort study. Lancet Neurol 2013; 12: 157-65.

Vincent A, Buckley C, Schott JM, et al. Potassium channel antibody-associated encephalopathy: a potentially immunotherapy-responsive form of limbic encephalitis. Brain 2004; 127: 701-12.

Zuliani L, Graus F, Giometto B, Bien C, Vincent A. Central nervous system neuronal surface antibody associated syndromes: review and guidelines for recognition. J Neurol Neurosurg Psychiatry 2012; 83: 638-45. 